\title{
Supersymmetric QCD from noncommutative geometry
}

\author{
Thijs van den Broek ${ }^{\mathrm{a}, \mathrm{b}, *}$, Walter D. van Suijlekom ${ }^{\mathrm{a}}$ \\ a Institute for Mathematics, Astrophysics and Particle Physics, Faculty of Science, Radboud University Nijmegen, Heyendaalseweg 135, 6525 AJ Nijmegen, The Netherlands \\ ${ }^{\mathrm{b}}$ National Institute for Subatomic Physics, Science Park 105, 1098 XG Amsterdam, The Netherlands
}

\section{A R T I C L E I N F O}

\section{Article history:}

Received 21 January 2011

Received in revised form 17 March 2011

Accepted 22 March 2011

Available online 1 April 2011

Editor: M. Cvetič

\section{Keywords:}

Noncommutative geometry

Supersymmetry

Supersymmetric QCD

Einstein-Yang-Mills

\begin{abstract}
A B S T R A C T
We derive supersymmetric quantum chromodynamics from a noncommutative spin manifold. We extend the model of Chamseddine and Connes that leads to the Einstein-Yang-Mills action and apply the spectral action principle to derive the Lagrangian of supersymmetric QCD, including soft supersymmetry breaking (negative sign) mass terms for the squarks. We find that these results are in good agreement with the physics literature.
\end{abstract}

(C) 2011 Elsevier B.V. All rights reserved.
Physics and geometry share a fruitful history. The most prominent example of that is Einstein's General Relativity, described in the language of Riemannian geometry. Noncommutative geometry [1] provides, amongst others, a generalization of the latter. Over the past years it has emerged as a successful tool for deriving models in high-energy physics from geometrical principles. Its main appeal is that it unifies gauge theories with General Relativity. The prime result [2] (following [3,4], see also [5]) by Chamseddine, Connes and Marcolli, who continued on the path set out by Connes and Lott [6], is a geometrical derivation of the full Standard Model, coupled to gravity and automatically including the famous Higgs mechanism. On top of that, in the noncommutative description of the Standard Model there naturally appear relations between Standard Model parameters, allowing for experimentally testable predictions $[2, \S 5]$.

Ever since the early days of the field, there was an interest in the possible compatibility of noncommutative geometry and supersymmetry: can noncommutative geometry describe supersymmetric theories? This has so far led to some tentative results [7-10]. In this Letter we take a rather different approach. In a way fully compliant with the paradigm of noncommutative geometry we derive the action of the supersymmetric extension of QCD, the part of the Standard Model that describes quarks and gluons. This Letter

\footnotetext{
* Corresponding author at: Institute for Mathematics, Astrophysics and Particle Physics, Faculty of Science, Radboud University Nijmegen, Heyendaalseweg 135, 6525 AJ Nijmegen, The Netherlands.

E-mail addresses: T.vandenBroek@science.ru.nl (T. van den Broek), waltervs@math.ru.nl (W.D. van Suijlekom).
}

can be considered as the more conceptual counterpart of [14], in which focus lies primarily on the calculations and technical details.

\section{The Einstein-Yang-Mills system from a noncommutative manifold}

The best way to introduce the key ideas is to derive $S U(N)$ Yang-Mills theory, coupled to fermions in the adjoint representation, from a noncommutative manifold [4]. In order to understand what the latter means, it is important to make the step of first algebraizing ordinary Riemannian (spin) geometry. Given a compact Riemannian spin manifold $M$, say of dimension 4, (local) coordinate functions $x_{\mu}$ are continuous maps from $M$ to $\mathbb{R}$. This leads us to consider the algebra of continuous functions $C(M)$ instead of $M$ itself. Then, given the spin structure on $M$ we have a Hilbert space of square-integrable spinors, denoted by $L^{2}(S)$. There is an action of $C(M)$ on it by pointwise multiplication. Also, we have a Dirac operator $\not \partial:=i \gamma^{\mu}\left(\partial_{\mu}+\omega_{\mu}\right)$ on $L^{2}(S)$, where $\omega_{\mu}$ is the spin connection accounting for $M$ not being flat. The triple $\left(C(M), L^{2}(S), \not \partial\right)$ is an example of a so-called spectral triple, the basic device in noncommutative geometry [1]. We will not dwell on the mathematical properties that it satisfies, but work in the explicit examples of interest.

The chirality operator $\gamma_{5}$ defines a grading operator on $L^{2}(S)$ (since $\gamma_{5}^{2}=1$ ) and charge conjugation gives rise to an operator $J_{L^{2}(S)}$ on $L^{2}(S)$ which satisfies $J_{L^{2}(S)}^{2}=-1$ (note the Euclidean signature).

Up to now, nothing noncommutative has happened: the algebra of functions on $M$ is still commutative. We would like to change 
that, so let us instead consider matrix-valued functions on $M$. In other words, the coordinates on $M$ become matrix-valued and the corresponding noncommutative algebra is $C\left(M, M_{N}(\mathbb{C})\right)$, or, which is the same, $C(M) \otimes M_{N}(\mathbb{C})$. Here $M_{N}(\mathbb{C})$ denotes the $N \times N$ complex matrices. Accordingly, consider matrix-valued spinors (i.e. in the adjoint representation), giving the Hilbert space $\mathcal{H}=L^{2}(S) \otimes$ $M_{N}(\mathbb{C})$. The action of $C\left(M, M_{N}(\mathbb{C})\right)$ on this Hilbert space is the combination of pointwise multiplication on the first entry of the tensor product with matrix multiplication on the second. We leave the Dirac operator as it is, acting only on the spinorial part: $D=\not \partial \otimes 1$. Similarly, a chirality operator is given by $\gamma:=\gamma_{5} \otimes 1$, whereas charge conjugation is now $J=J_{L^{2}(S)} \otimes(\cdot)^{\dagger}$, where the latter part stands for taking the Hermitian conjugate of a matrix. In analogy with the above triple for the Riemannian spin manifold $M$, we consider

\section{$\left(C\left(M, M_{N}(\mathbb{C})\right), L^{2}(S) \otimes M_{N}(\mathbb{C}), D\right)$.}

This can be thought of as describing the product $M \times F$ of spacetime $M$ with a noncommutative space $F$. The latter is described by a finite-dimensional algebra - in this case $M_{N}(\mathbb{C})-$ and will be referred to as the finite part. Consequently, a spectral triple defined on such a finite-dimensional algebra is called a finite spectral triple.

Let us describe how to derive usual $S U(N)$-Yang-Mills theory from this noncommutative manifold. As usual, one considers unitary transformations of the constituents of our theory, which amounts to

$D \mapsto U D U^{\dagger} ; \quad \gamma \mapsto U \gamma U^{\dagger} ; \quad J \mapsto U J U^{\dagger}$

for some unitary operator $U$ on the Hilbert space $L^{2}(S) \otimes M_{N}(\mathbb{C})$. Here, we restrict to unitary operators of the form $U=u J u J^{\dagger}$ where $u$ is a unitary element in the algebra $C\left(M, M_{N}(\mathbb{C})\right)$. The expression $J u J^{\dagger}$ is right multiplication with $u^{\dagger}$. In this case, the above transformation leaves $\gamma$ and $J$ invariant, but changes $D$ to $D+A+J A J^{\dagger}=: D_{A}$, i.e. left and right multiplication with $A=u\left[D, u^{\dagger}\right] \equiv \gamma^{\mu} A_{\mu}$. Here $A_{\mu}$ is a pure gauge, $i u(N)$-valued function on $M$. One derives that the so-called fluctuated Dirac operator (the covariant derivative) is

$D_{A}=i \gamma^{\mu}\left[\left(\partial_{\mu}+\omega_{\mu}\right) \otimes 1+1 \otimes \mathbb{A}_{\mu}\right]$,

where $\mathbb{A}_{\mu}:=-i$ ad $A_{\mu}\left(\operatorname{ad}\left(A_{\mu}\right)\right.$ means taking the commutator with $A_{\mu}$ ) is skew-Hermitian due to the self-adjointness of $A_{\mu}$ and is thus $s u(N)$-valued, but not necessarily pure gauge. The gauge group thus is $C(M, U(N))$, consisting of the unitary elements in $C\left(M, M_{N}(\mathbb{C})\right)$. Note that the non-abelian nature of this group is a direct consequence of the noncommutativity in $C\left(M, M_{N}(\mathbb{C})\right.$ ).

It is then natural to seek for gauge invariant functionals of the gauge fields and the simplest formula one could come up with is the trace of $D_{A}^{2}$. This is, however, ill-defined (because $D_{A}^{2}$ is not traceclass) and incomplete from the physics point of view (we would be missing quartic interactions), but the following spectral action $[4,11]$ does work:

$S_{b}[A]:=\operatorname{Tr} f\left(D_{A} / \Lambda\right)$.

Here $\Lambda$ is a cut-off scale and $f$ some positive, even function that should in the end be fixed by comparison with known results. The cut-off parameter $\Lambda$ can be used to obtain an asymptotic expansion for the spectral action; the terms with a positive power of $\Lambda$ as a coefficient are then the physically relevant ones. The fermionic action is given by

$S_{f}[A, \psi]:=\left\langle\psi, D_{A} \psi\right\rangle$,

where $\langle\cdot, \cdot\rangle$ denotes the inner product on the Hilbert space $\mathcal{H}$. It turns out ([4], see also [5, Sect. 11.4]) that the spectral action has an asymptotic expansion (as $\Lambda \rightarrow \infty$ ) of the form:
Table 1

The number of degrees of freedom for both fields appearing in the Einstein-YangMills system.

\begin{tabular}{llllll}
\hline & \multicolumn{2}{c}{ Currently } & & \multicolumn{2}{c}{ Needed } \\
\cline { 2 - 3 } \cline { 6 - 6 } & Continuous & Finite & & Continuous & Finite \\
\hline Bosons & 4 & $N^{2}-1$ & & $N^{2}-1$ \\
Fermions & 8 & $2 N^{2}$ & & 4 & $N^{2}-1$ \\
\hline
\end{tabular}

$S_{b}[A]=\frac{1}{4 \pi^{2}} \int_{M} \mathcal{L}_{b}(g, A)+\mathcal{O}\left(\Lambda^{-2}\right)$,

with Lagrangian

$$
\begin{aligned}
\mathcal{L}_{b}(g, A)= & 2 f_{4} \Lambda^{4} N^{2}+\frac{N^{2}}{6} f_{2} \Lambda^{2} R+\frac{f(0) N^{2}}{1440}\left[5 R^{2}-8 R_{\mu \nu} R^{\mu \nu}\right. \\
& \left.-7 R_{\mu \nu \rho \sigma} R^{\mu \nu \rho \sigma}\right]-\frac{f(0)}{6} \operatorname{Tr}\left(\mathbb{F}_{\mu \nu} \mathbb{F}^{\mu \nu}\right),
\end{aligned}
$$

where $\mathbb{F}_{\mu \nu}$ is the field strength of $\mathbb{A}_{\mu}, R_{\nu \rho \sigma}^{\mu}$ the Riemann curvature tensor of $M$ and $f_{2,4}$ the second and fourth moments of $f$ respectively. This expression contains both the Einstein-Hilbert action of General Relativity and the Yang-Mills action of an $S U(N)$ gauge field. Since the term $\left\langle\psi, D_{A} \psi\right\rangle$ accounts for the fermionic propagator and interactions of the fermion $\psi$ with the gauge field, the sum

$S[A, \psi]:=\operatorname{Tr} f\left(D_{A} / \Lambda\right)+\left\langle\psi, D_{A} \psi\right\rangle$

gives the full action of the Einstein-Yang-Mills system plus terms of order $\Lambda^{-2}$. The gauge group $C(M, S U(N))$ acts on the gauge potential $A$ and on $\psi$ in the adjoint representation.

It is essentially this set up that leads to a description of the Standard Model [2-5].

\section{Supersymmetry in noncommutative geometry}

We would like to obtain a realization of supersymmetry for the Einstein-Yang-Mills system in the framework of noncommutative geometry. This can be seen as a first step towards supersymmetric QCD, since it essentially describes gluons and gluinos. The possibility of such a supersymmetry was suggested in [4]. A necessary condition for supersymmetry is that we have an equal number of bosonic and fermionic degrees of freedom. This is not the case yet. Indeed, both in the spinorial as in the finite part the fermionic degrees of freedom exceed those of the bosons: a spinor $\psi(x)$ has eight real (four complex) degrees of freedom whereas the continuous part of the gauge potential has only four: $A_{\mu}, \mu=1, \ldots, 4$, and on the finite part we automatically got a reduction for the bosons from $2 N^{2}$ to $N^{2}-1$ real degrees of freedom. The degrees of freedom are summarized in Table 1. In this Euclidean setup $J^{2}=-1$, so no Majorana fermions exist [12] and we have to use Weyl spinors instead. In contrast to [2] we cannot restrict to Weyl fermions by altering the inner product, for $J \gamma \neq-\gamma J$ here. Instead we employ a scheme due to Van Nieuwenhuizen and Waldron [13]; we both relax the reality condition on the action and Wick rotate the spinors $\psi, \bar{\psi}$ and gamma-matrices, as appearing in a Minkowskian setup, to the Euclidean case, resulting in spinors $\psi, \chi$ of opposite chirality. The path integral is insensitive to such a rotation since the system still contains two fermionic variables ( $\psi$ and $\chi$ instead of $\psi$ and $\bar{\psi}$ ). To summarize, the solution is thus to take as the fermionic part of the action

$S_{f}[A, \psi, \chi]:=\left\langle\chi, D_{A} \psi\right\rangle ; \quad \psi \in \mathcal{H}^{+}, \chi \in \mathcal{H}^{-}$,

which is the Euclidean counterpart of the action for $\bar{\psi}$ and $\psi$ in Minkowskian space. 
For the finite part we obtain the reduction from $M_{N}(\mathbb{C})$ to $\mathfrak{s u}(N)$ by first using the fact that any complex matrix can be written as the sum of a Hermitian and an anti-Hermitian matrix, i.e. it is the complexification of $M_{N}(\mathbb{C})$, followed by splitting a fermion into a trace and a traceless part: $\tilde{\psi}=\operatorname{Tr} \tilde{\psi}+\psi \in$ $L^{2}(S) \otimes(\mathfrak{u}(1) \oplus \mathfrak{s u}(N))$. These components fully decouple in the action and the trace-part lacks any gauge interactions:

$S_{f}[A, \tilde{\psi}, \tilde{\chi}]=\langle\operatorname{Tr} \tilde{\chi}, D \operatorname{Tr} \tilde{\psi}\rangle+\left\langle\chi, D_{A} \psi\right\rangle$.

We can therefore discard it from the theory, thus retaining only $S U(N)$-valued Weyl spinors.

Thus, consider the action $S[A, \psi, \chi]$ that results from (5) in the case of the Einstein-Yang-Mills action, but now with two $S U(N)$ valued Weyl spinors $\psi$ and $\chi$. Write the fermionic part

$S_{f}[A, \psi, \chi]=\left\langle\chi, D_{A} \psi\right\rangle=\int_{M} \operatorname{Tr}_{F}\left(\chi, D_{A} \psi\right)$,

in terms of the spinorial inner product $(\cdot, \cdot)$ and a trace $\operatorname{Tr}_{F}$ over the finite part.

From here on $\epsilon_{ \pm}$will denote a pair of $\gamma^{5}$ eigenspinors that are singlets of the gauge group and vanish covariantly: $\left(\partial_{\mu}+\right.$ $\left.\omega_{\mu}\right) \epsilon_{ \pm}=0$. For the gauge field $A$, and spinors $\psi, \in \mathcal{H}^{+}, \chi \in \mathcal{H}^{-}$ we define $\delta A$ and $\delta \psi \in \mathcal{H}^{+}, \delta \chi \in \mathcal{H}^{-}$by

$\delta A:=\gamma^{\mu}\left[\left(\epsilon_{-}, \gamma_{\mu} \psi\right)+\left(\chi, \gamma_{\mu} \epsilon_{+}\right)\right]$and

$\delta \psi, \delta \chi:=-\frac{i f(0)}{6 \pi^{2}} F \epsilon_{+,-}$

where $F \equiv \gamma^{\mu} \gamma^{\nu} F_{\mu \nu}^{a} \otimes T_{a}, F_{\mu \nu}=\partial_{\mu} A_{\nu}-\partial_{\nu} A_{\mu}+\left[A_{\mu}, A_{\nu}\right]$. One can check [14] that the action (5) of the Einstein-Yang-Mills system is invariant under the supersymmetry transformations (6) for at least all physically relevant terms in the expansion of the spectral action.

We particularize to $N=3$ since we are after a supersymmetric version of QCD. In order to accommodate not only gluons and gluinos, but the quarks and antiquarks as well, we enlarge the finite part of the Hilbert space by adding two copies of $\mathbb{C}^{3}$ to it. The superpartners of the quarks - the squarks - are scalars and in the paradigm of noncommutative geometry should always be generated by the Dirac operator of a finite spectral triple, as is the case for the Higgs boson in the Standard Model [2]. This suggests that we consider the noncommutative manifold described by the triple:

$\mathcal{A}=C\left(M, M_{3}(\mathbb{C})\right)$

$\mathcal{H}=L^{2}(S) \otimes\left(\mathbb{C}^{3} \oplus M_{3}(\mathbb{C}) \oplus \mathbb{C}^{3}\right) \ni\left(\psi_{q}, \psi_{g}, \psi_{\bar{q}}\right) ;$

$D=\partial_{M} \otimes 1+\gamma_{5} \otimes D_{F}$.

In order for the quarks and squarks to be in the same representation of the gauge group (as is required for supersymmetry) there is only one possible form for the finite Dirac operator:

$D_{F}:=\left(\begin{array}{ccc}0 & d & 0 \\ d^{\dagger} & 0 & e^{\dagger} \\ 0 & e & 0\end{array}\right)$,

i.e. it must map gluinos to (anti)quarks and vice versa according to $d\left(\psi_{g}\right):=\psi_{g} v$ and $e\left(\psi_{g}\right):=\psi_{g}^{t} \bar{v}$, parametrized by some $v \in \mathbb{C}^{3}$. Also, $\mathcal{A}$ acts on the left of $\mathcal{H}$ as $a\left(\psi_{q}, \psi_{g}, \psi_{\bar{q}}\right)=\left(a \psi_{q}, a \psi_{g}, \psi_{\bar{q}}\right)$, where pointwise multiplication on the spinorial part is understood. There is a chirality operator given by $\gamma=\gamma_{5} \otimes 1$ and charge conjugation now becomes

$J\left(\psi_{q}, \psi_{g}, \psi_{\bar{q}}\right)=\left(J_{L^{2}(S)} \psi_{\bar{q}}, J_{L^{2}(S)} \psi_{g}^{\dagger}, J_{L^{2}(S)} \psi_{q}\right)$
In other words, on the $M_{3}(\mathbb{C})$-part it is the same as that for the Einstein-Yang-Mills system, but it will exchange the two copies of $\mathbb{C}^{3}$ (the quark and antiquark), followed by charge conjugation on $L^{2}(S)$.

We find that at each $x \in M$ the inner fluctuations $D_{A}=D+A+$ $J A J^{\dagger}$ are parametrized by an $S U(3)$-gauge potential $\mathbb{A}_{\mu}(x)$ and a $\mathbb{C}^{3}$-valued function $\tilde{q}(x)$ :

$D_{A}=\not \partial \otimes 1+i \gamma^{\mu} \mathbb{A}_{\mu}+\gamma_{5} \otimes D_{\tilde{q}}$

with the operator $D_{\tilde{q}}$ given by

$D_{\tilde{q}}\left(\psi_{q}, \psi_{g}, \psi_{\bar{q}}\right)=\left(\psi_{g} \tilde{q}, \psi_{q} \overline{\tilde{q}}^{t}+\tilde{q} \psi_{\bar{q}}^{t}, \psi_{g}^{t} \overline{\tilde{q}}\right)$.

We will identify $\tilde{q}$ and $\overline{\tilde{q}}$ as the squark and anti-squark, respectively. As before, $\mathbb{A}$ will be the gluon, and $\psi_{g} \in L^{2}(S) \otimes M_{3}(\mathbb{C})$ the gluino. This nomenclature is justified by the fact that the quarks and squarks are in the same representation of the gauge group, as are the gluons and gluinos.

The corresponding spectral action is determined to be (see [14] for the technical details):

$$
\begin{aligned}
S_{b}[\tilde{q}, A]= & S_{b}^{\prime}[A]+\int_{M}\left[-\frac{6 f_{2}}{\pi^{2}} \Lambda^{2}|\tilde{q}(x)|^{2}+\frac{f(0)}{4 \pi^{2}}\right. \\
& \left.\times\left(8|\tilde{q}(x)|^{4}+6\left|D_{\mu} \tilde{q}(x)\right|^{2}-3 R|\tilde{q}(x)|^{2}\right)\right]+\mathcal{O}\left(\Lambda^{-2}\right)
\end{aligned}
$$

where $S_{b}^{\prime}[A]$ is the action of the Einstein-Yang-Mills system (cf. (4)). The fermionic action becomes

$$
\begin{aligned}
S_{f}[ & \left.A, \psi_{q}, \psi_{g}, \chi_{g}, \psi_{\bar{q}}\right] \\
\equiv & \left\langle\left(\psi_{q}, \chi_{g}, \psi_{\bar{q}}\right), D_{A}\left(\psi_{q}, \psi_{g}, \psi_{\bar{q}}\right)\right\rangle \\
= & \left\langle\psi_{q},(\not \partial+A) \psi_{q}\right\rangle+\left\langle\chi_{g},(\not \partial+\mathbb{A}) \psi_{g}\right\rangle \\
& +\left\langle\psi_{\bar{q}},(\not \partial+\bar{A}) \psi_{\bar{q}}\right\rangle+\left\langle\psi_{q}, \psi_{g} \tilde{q}\right\rangle+\left\langle\chi_{g} \tilde{q}, \psi_{q}\right\rangle \\
& +\left\langle\chi_{g}^{t} \overline{\tilde{q}}, \psi_{\bar{q}}\right\rangle+\left\langle\psi_{\bar{q}}, \psi_{g}^{t} \overline{\tilde{q}}\right\rangle,
\end{aligned}
$$

in which we recognize additional quark-squark-gluino and gluongluino-gluino interactions.

Upon switching to flat Minkowski space these results are seen to be in excellent agreement with the literature on the Minimally Supersymmetric Standard Model (e.g. [15,16]); all interactions are present and their form is precisely the same. Not for all terms, however, do the coefficients match exactly. Some of these coefficients depend on the dimension of the representations in the finite Hilbert space and thus differ from the value one would obtain from a description of the full MSSM. Addressing this question is part of future research.

One observation that we cannot refrain from making is that the sum $S_{b}+S_{f}$ is in fact not supersymmetric; there appear (negative sign) squark mass terms as allowed in soft supersymmetry breaking (see e.g. [16]). We consider the presence of these terms as a merit of the above model, leaving the question open whether a soft susy-breaking mechanism, responsible for these terms, can be found within noncommutative geometry. Possibly, one of the noncommutative manifolds that appear in the classification of [17] will describe the supersymmetric theory with a spontaneous supersymmetry breaking mechanism.

\section{References}

[1] A. Connes, Noncommutative Geometry, Academic Press, San Diego, 1994.

[2] A.H. Chamseddine, A. Connes, M. Marcolli, Adv. Theor. Math. Phys. 11 (2007) 991.

[3] A.H. Chamseddine, A. Connes, Phys. Rev. Lett. 77 (1996) 4868. 
[4] A.H. Chamseddine, A. Connes, Commun. Math. Phys. 186 (1997) 731.

[5] A. Connes, M. Marcolli, Noncommutative Geometry, Quantum Fields and Motives, AMS, Providence, 2008.

[6] A. Connes, J. Lott, Nucl. Phys. B (Proc. Suppl.) 18 (1991) 29.

[7] F. Hussain, G. Thompson, Phys. Lett. B 260 (1991) 359.

[8] F. Hussain, G. Thompson, Phys. Lett. B 265 (1991) 307.

[9] A.H. Chamseddine, Phys. Lett. B 332 (1994) 349.

[10] W. Kalau, M. Walze, J. Geom. Phys. 22 (1997) 77.
[11] A. Connes, Commun. Math. Phys. 182 (1996) 155.

[12] J. Schwinger, Phys. Rev. 115 (1959) 721.

[13] P. van Nieuwenhuizen, A. Waldron, Phys. Lett. B 389 (1996) 29.

[14] T. van den Broek, W. van Suijlekom, Commun. Math. Phys. 303 (2011) 149

[15] S. Kraml, Stop and sbottom phenomenology in the MSSM, hep-ph/9903257, 1999.

[16] D.J.H. Chung, et al., Phys. Rep. 407 (2005) 1.

[17] A.H. Chamseddine, A. Connes, J. Geom. Phys. 58 (2008) 38. 